\title{
ASPECTS ISOTOPIQUES DU CYCLE ATMOSPHÉRIQUE DE L'EAU : MODIFICATIONS APPORTÉES PAR DES VARIATIONS DE CLIMAT A GRANDE ÉCHELLE
}

\author{
J. C. DUPLESSY \\ Centre des Faibles Radioactivités. Laboratoire mixte CNRS/CEA, \\ 91190 Gif-sur-Yvette, France
}

\begin{abstract}
Résumé. - Le cycle atmosphérique de l'eau est profondément affecté par les variations du climat au cours des temps géologiques. Cet article montre comment les aspects isotopiques de ce cycle sont utilisés pour l'étude des climats anciens.
\end{abstract}

\begin{abstract}
Atmospheric water cycle is strongly depending on climatic variations which occur during geologic times. This paper shows the isotopic effects of climatic variations and their uses in paleoclimatological studies.
\end{abstract}

Le climat de la terre a subi d'importantes fluctuations depuis $10^{6}$ ans. Nous sommes actuellement dans une phase chaude. Lorsque s'établit une période froide, le développement de vastes calottes glaciaires à la surface des continents soustrait du cycle atmosphérique une importante quantité d'eau.

Les conséquences de ce phénomène constituent les bases de la paléoclimatologie isotopique. Depuis une vingtaine d'années ce domaine de recherche a subi d'importants développements et nous nous limiterons ici à donner les grands sujets d'étude avec une bibliographie permettant au lecteur intéressé de se documenter.

1. Les variations du niveau de la mer. - C'est une conséquence immédiate du bilan des masses d'eau à l'état liquide et sous forme solide. Le développement des calottes glaciaires qui, à son maximum, il y a 18000 ans couvraient tout le nord de l'Amérique et de l'Europe [1] a entraîné une diminution de l'ordre de $3 \%$ du volume d'eau à l'état liquide; ceci s'est traduit par une baisse du niveau de la mer d'environ 120 mètres [2].

2. Le déséquilibre du budget des isotopes de l'oxygène et de l'hydrogène. - Les pluies et neiges condensées étant d'autant plus pauvres en isotopes lourds $\mathrm{O}^{18}$ et $\mathrm{D}$ que la température de condensation est plus basse (voir l'article de L. Merlivat dans ce numéro du Journal de Physique, page 11), il en résulte que les précipitations sont d'autant plus pauvres en isotope lourd qu'elles se produisent aux hautes latitudes (Fig. 1).

Une première application paléoclimatique est la suivante : si le climat devient plus froid, en un même lieu les précipitations se condensent en moyenne à

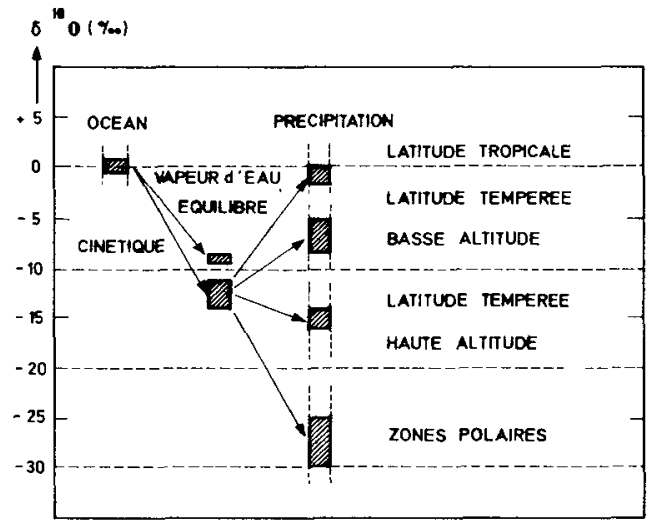

Fig. 1. - Diagramme schématique représentant les variations du rapport ${ }^{18} \mathrm{O} /{ }^{16} \mathrm{O}$ des précipitations aux différentes latitudes. Etant extrêmement faibles, les variations du rapport ${ }^{18} \mathrm{O} /{ }^{16} \mathrm{O}$ sont exprimées dans l'unité $\delta$ définie par la relation

$$
\delta^{18} \mathrm{O}=\left[\frac{\left({ }^{18} \mathrm{O} /{ }^{16} \mathrm{O}\right) \text { échantillon }}{\left({ }^{18} \mathrm{O} /{ }^{16} \mathrm{O}\right) \text { standard }}-1\right] \times 1000 .
$$

Une valeur de $\delta^{18} \mathrm{O}$ positive exprime donc que l'échantillon est plus riche en oxygène 18 que le standard et inversement une valeur négative de $\delta^{18} \mathrm{O}$ exprime que l'échantillon est plus pauvre en oxygène 18 que le standard. Le standard utilisé dans ce diagramme est l'eau de mer moyenne.

une température plus faible et sont donc plus pauvres en isotopes lourds. Duplessy et al. [3, 4] ont montré que ces variations sont enregistrées dans les variations du rapport ${ }^{18} \mathrm{O} /{ }^{16} \mathrm{O}$ des concrétions des cavernes. Les figures 2 et 3 donnent deux exemples d'application de cette méthode. Elles permettent de mettre en évidence en particulier le fait que d'importants changements de climat peuvent se produire très rapidement (quelques siècles seulement). 


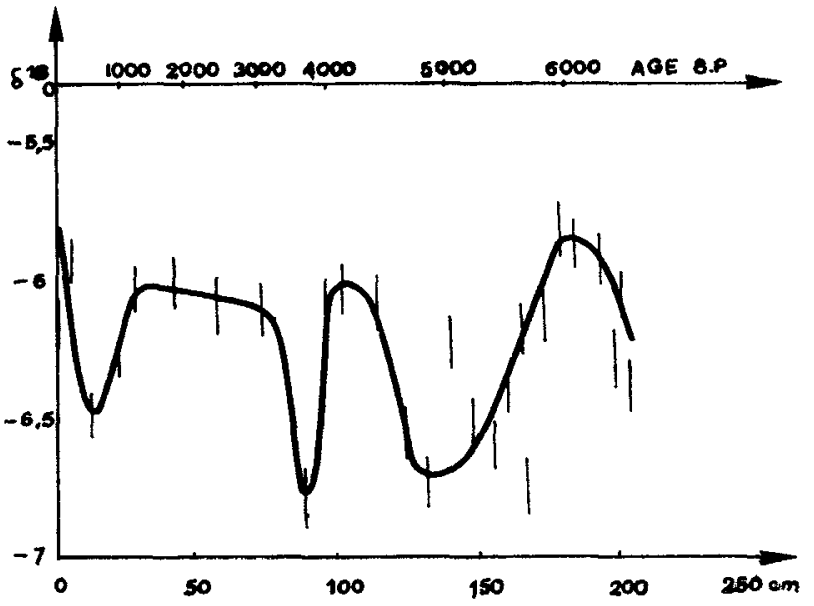

FIG. 2. - Variations du rapport ${ }^{18} \mathrm{O} /{ }^{16} \mathrm{O}$ d'une concrétion de l'Aven d'Orgnac au cours des 6500 dernières années [3]. Plus le carbonate est pauvre en oxygène $18\left(\delta^{18} \mathrm{O}\right.$ très négatif) plus l'eau de pluie au moment de la formation de la concrétion était pauvre en oxygène 18 et plus le climat était froid. L'amplitude maximale des fluctuations représente une variation de température de $1,5^{\circ} \mathrm{C}$.

Une seconde application paléoclimatique provient du fait que les calottes glaciaires sont très pauvres en isotopes lourds [5]. La mer se retrouve donc relativement d'autant plus riche en isotopes lourds que les calottes glaciaires sont plus développées sur les continents. C. Emiliani [6], J. C. Duplessy et al. [7], Shackleton et Opdyke [8] ont montré que le rapport ${ }^{18} \mathrm{O} /{ }^{16} \mathrm{O}$ de l'océan se reflète dans celui des carbonates qui s'y précipitent. En mesurant les variations de la composition isotopique des foraminifères fossiles prélevés dans les carottes océaniques il est alors possible de mesurer les variations du rapport ${ }^{18} \mathrm{O} /{ }^{16} \mathrm{O}$ de l'océan dans le passé et d'en déduire l'évolution du climat moyen du globe.

A titre d'exemple (Fig. 4) nous présentons l'analyse de la carotte MD 7304 prélevée dans l'océan Indien au cours de la campagne OSIRIS I du Marion

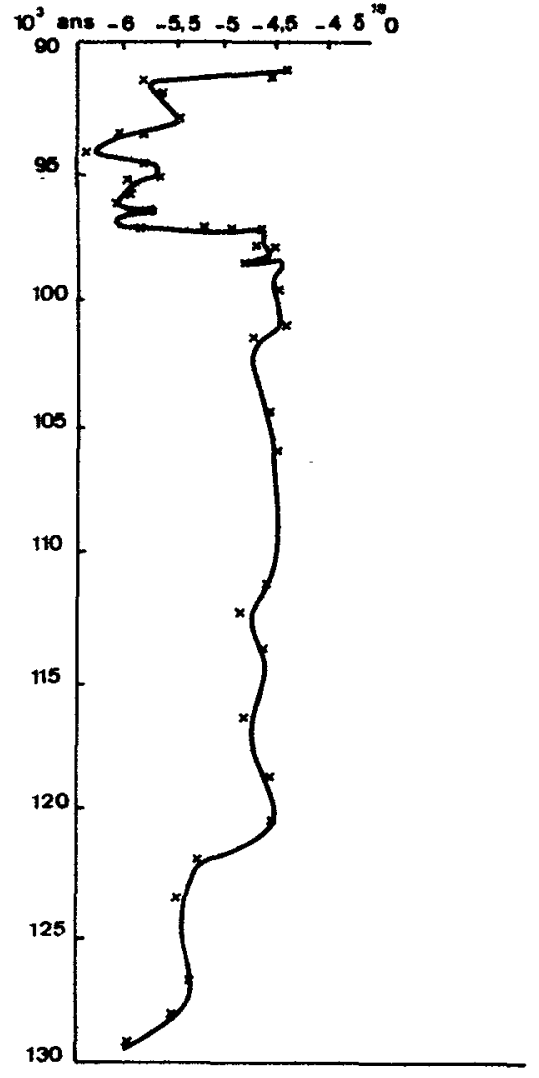

FIG. 3. - Variations du rapport ${ }^{18} \mathrm{O} /{ }^{16} \mathrm{O}$ d'une concrétion de l'Aven d'Orgnac entre 90000 et 130000 B.P. [4].

Dufresne, navire de relève des Terres Australes et Antarctiques Françaises.

La différence de composition isotopique entre l'océan et les glaces polaires dépendant de la circulation atmosphérique, la comparaison des rapports ${ }^{18} \mathrm{O} /{ }^{16} \mathrm{O}$ des glaces déposées dans le passé et de l'océan à la même époque permet de montrer que les vents sont en moyenne plus intenses pendant les périodes glaciaires [9].

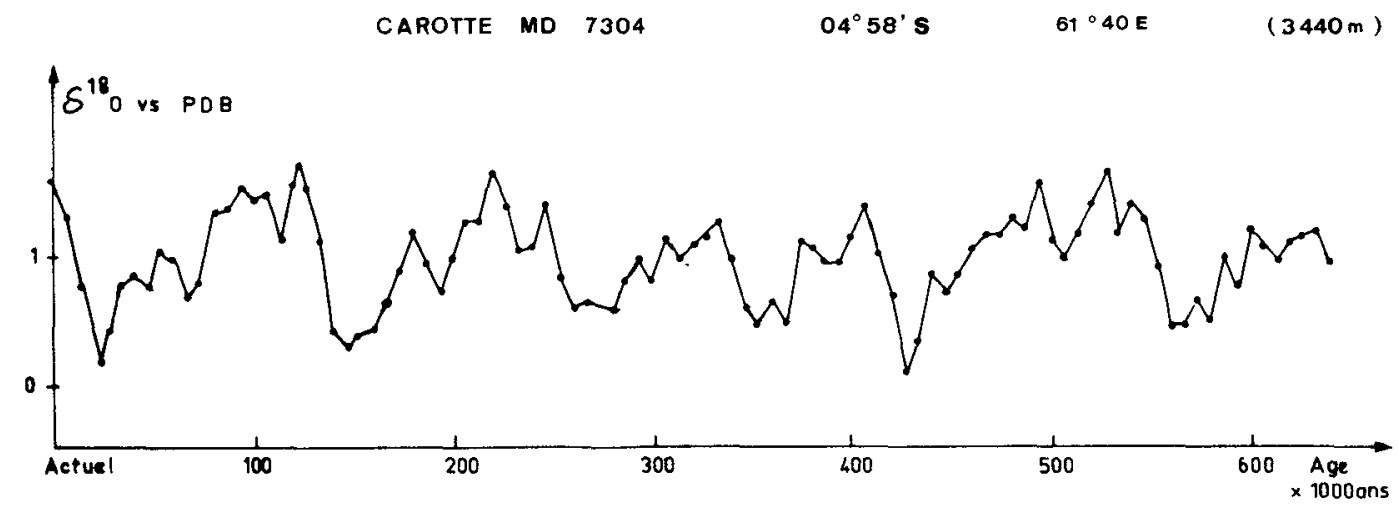

FIG. 4, - Variations du rapport ${ }^{18} \mathrm{O} /{ }^{16} \mathrm{O}$ des foraminifères pélagiques Globigerinoides sacculifer le long de la carotte MD 7304 . Les périodes climatiques chaudes (environ 1 tous les $100000 \mathrm{ans}$ ) sont caractérisées par des $\delta{ }^{18} \mathrm{O}$ négatifs qui représentent une fonte maximale des calottes glaciaires. 


\section{Bibliographie}

[1] FLINT, R. F., Glacial and Quaternary Geology (Wiley) 1971.

[2] Lalou, C., Duplessy, J. C. et NguYen, H. V., Rev. Geog. Phys. et Geol. Dyn. 13 (1971) 447.

[3] Labeyrie, J., Duplessy, J. C., Delibrias, G. and Letolle, R., Radioactive dating and Methods of Low-Level Counting, IAEA, 1957, 153.

[4] Duplessy, J. C., Labeyrie, J., Lalou, C. et Nguyen, H. V., Quat. Res. 1 (1971) 162.
[5] Dansgaard, W., Johnsen, S. J., Clausen, H. B. et LangWAY, C. C., Late Cenezoïc Glacial Ages, K. K. Turekian ed. (Yale University Press) 1971.

6] Emiliani, C., J. Geology 63 (1955) $\mathrm{n}^{\circ} 6$.

[7] Duplessy, J. C., Lalou, C. et Vinot, A. C., Science 168 (1970) 250.

[8] Shackleton, N. J. and Opdyke, N. D., Quat. Res. 3 (1973) 39.

[9] Hendy, C. H. and Wilson, A. T., Nature 234 (1971) 344. 